\title{
Design of Slew Bearing of FPSO System Based on Finite Element Analysis
}

\author{
Chao Wang \\ Automatic Control Engineering Department,LiaoNing Mechatronics College, Dandong, LiaoNing , \\ China \\ 210-wangchao@163.com
}

\begin{abstract}
Keywords: finite element analysis;FPSO;slew bearing; design;
Abstract. Make the slew bearing for object of study which use of petroleum machinery.According to the requirements of customers, design the basic structure of slew bearing, Set up 3D-model of the slew bearing in the Solidworks. Through the appropriate simplification of the model, the slew bearing are properly loaded in the finite element analysis software Solidworks and ANSYS, and the stress analysis results of the raceway and bolt installation holes of the slew bearing are obtained. The analysis results provide some reference for the optimal design of slewing bearings, and thus reduce the cost of the design and manufacture of turntable bearings.
\end{abstract}

\section{Introduction}

The slew bearing is a large rolling bearing, which is often used in the yaw and pitch parts of the wind turbine and the rotating part of the engineering machinery. Ordinary rolling bearings mainly bear axial force and radial force, while slew bearing is applied on large mechanical equipment, so it bears axial force and overturning moment when it takes a small amount of radial load ${ }^{[1]}$. With the increasing proportion of green and lightweight in host design, more and more hosts require non-standard slew bearings, which puts forward higher requirements for the design of slew bearing.

Slewing bearing used in marine petroleum machinery FPSO, in the sea environment, safety and accuracy of bearing system has a very high demand.This slew bearing is combined with the experience method and the finite element analysis method. In using the experience method to design the basic structure of slewing bearings, slewing bearing on the static analysis through the finite element analysis method, improve the design efficiency of slewing bearings, reducing the cost of design and manufacture of the bearing, the bearing to meet the requirements of the carrying capacity, but also conform to the green requirements, at the same time, provide a reference according to the design and improvement of other bearings.

\section{Application environment of slew bearing}

The slew bearing is applied to the liquid swivel ring of offshore oil machinery. Liquid swivel ring is installed below or above the level, while the liquid swivel ring is moored to FPSO, in the influence of wind and ocean currents generated under reciprocating irregular rotation, and then drive the liquid ring rotation, so that the bearing inner ring and the outer ring has relative motion ${ }^{[2]}$, so the bearing design requirements are very high.

After testing, the slew bearing bears the axial force, the radial force and the overturning moment, and the external environment temperature: $0 \sim 90^{\circ} \mathrm{C}$, speed: $2 \mathrm{r} / \mathrm{d}$. At the same time, the bearing has a good seal, the design life : 15 years, the safety factor: 2 , tolerance grade: $\mathrm{P} 5$, the deformation of the end face is less than $0.005 \mathrm{~mm}$.

\section{Selection of type for slew bearing}

There are two main types of slew bearings: ball type (single row, double row) and roller type (cross roller, three-row roller). The bearing capacity of ball bearing is relatively low. The roller of cross roller roller bearing is prone to edge effect when it contacts with raceway, so it is less applied at present ${ }^{[3]}$. 
The load provided by customer is mainly axial force and overturning moment. The rotary bearing is connected to FPSO through the bolts of inner or outer ring. Therefore, use three-row roller slew bearing(Inner and outer ring without teeth ) comprehensive above factors

\section{Material and the basic design of structure for slew bearing}

The slew bearing ring adopts $42 \mathrm{CrMo}$, tempering treatment and hardness: 240 290HB.

Surface hardening is achieved on the raceway, and the hardness is 54 60HRC. The depth of the effective hardening layer is required: the axial direction is not less than $5 \mathrm{~mm}$, and the radial is not less than $3 \mathrm{~mm}$.

The rolling body of the slew bearing adopts the GCr15SiMn, which is separated by the cast aluminum isolation ring to ensure that each rolling body is uniformly subjected to force.

The slew bearing is filled with grease of Fuchs Gleitmo $585 \mathrm{~K}$, which can effectively reduce the rotational resistance moment and extend the service life of the slew bearing.

In order to prevent the leakage of grease and Invasion of impurities, two non-standard $\mathrm{O}$ ring seals were adopted at the two outer ring connections.

The inner and outer ring is sealed with a new sea ${ }^{[4]}$. This seal has a compound lip, which can improve the sealing effect ${ }^{[5]}$. The basic structure of the slew bearing is shown in Figure 1.

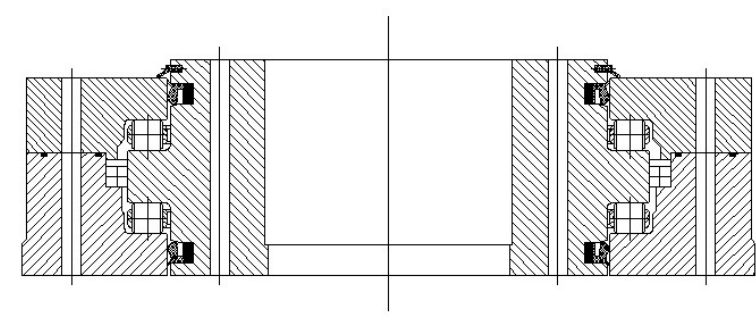

Fig.1 The Figure of Slew Bearing Structure

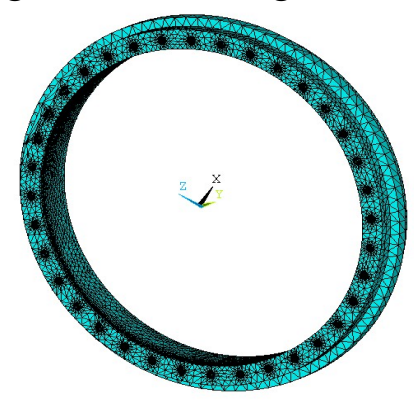

Fig.2 The Gridding Modle of Slew Bearing

\section{Stress analysis of slew bearing}

The load of the slew bearing is applied to the hole wall of the bolt mounting hole through the bolt of the circular direction of the turntable bearing. Therefore, the stress analysis of the inner ring bolt hole wall of the slew bearing is carried out.

First, the finite element analysis model of slew bearing is seted up by Solidworks. Then, the data interface is used to import the model into the ANSYS.

\section{Stress analysis of bolt mounting hole in slew bearing ${ }^{[6]}$}

\section{Grid division of slew bearing}

The gridding of the slew bearing is divided by Solid92 unit, which is the 10 node tetrahedral element of the three-dimensional entity, and the two unit with the middle node. Secondly, the modulus of elasticity of $42 \mathrm{CrMo}(2.12 \mathrm{e} 11)$ and Poisson's ratio (0.28) are input. Finally, the finite element mesh model obtained by the free mesh method, as shown in Figure 2.

\section{Loading for slew bearing model}

According to the actual working condition of the slew bearing, the contact surface of the radial roller is fully constrained. The load of the maximum destructive force of the rotating disk bearing is loaded equivalent, and the bearing force is at this time: $495 \mathrm{KN}$.

In the process of software analysis, the equivalent pressure of the slew bearing is loaded on the half side of the installation hole. The pressure of the slew bearing: $\mathrm{P}=131.2 \mathrm{Mpa}$. The constraints and loading diagrams are shown in Figure 3.

\section{Finite element analysis of slew bearing}

After analyzing and post-processing,we extract the nodal stress cloud diagram of the model from the calculation results, as shown in Figure 4, and the element stress is shown in Figure 5. 


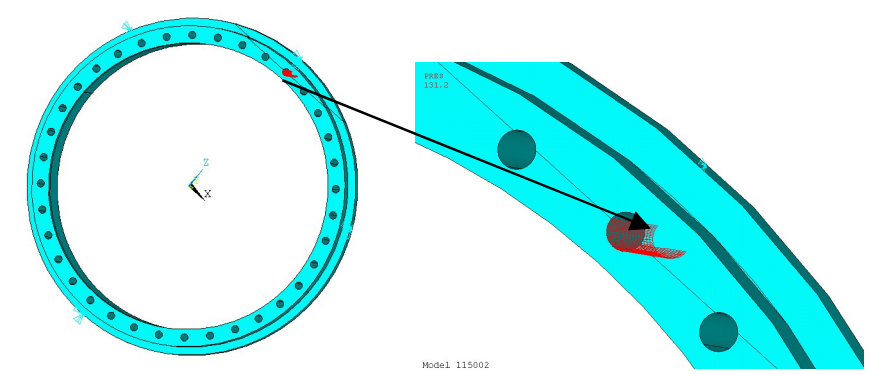

Fig.3 The Figure of Slew Bearing Bounded and Loaded

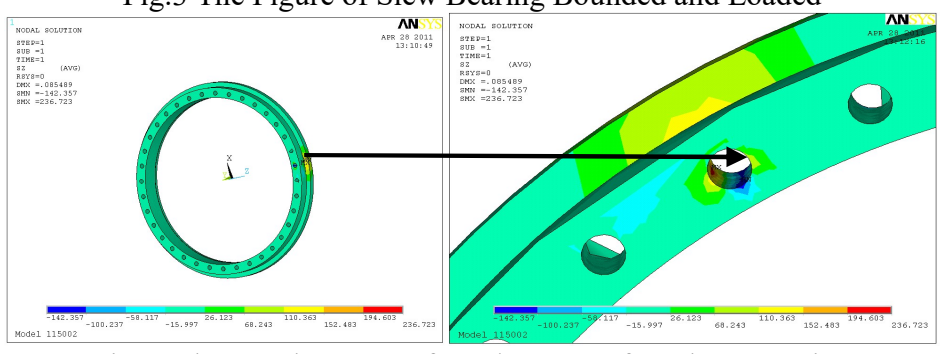

Fig.4 The Nephogram of Node Stress for Slew Bearing

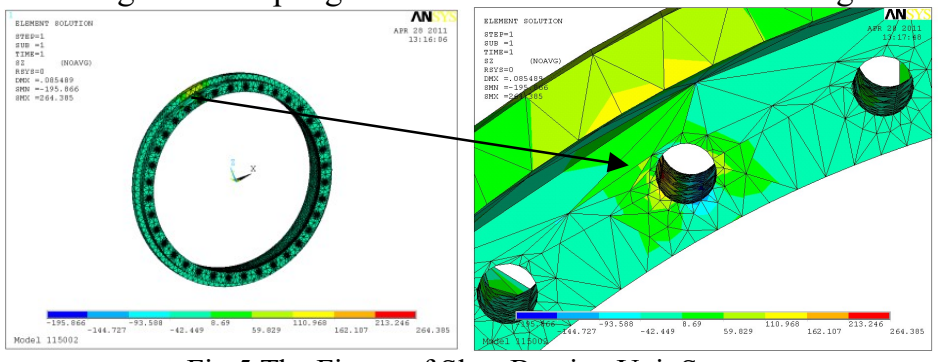

Fig. 5 The Figure of Slew Bearing Unit Stress

From the analysis results, it can be seen that the equivalent stress of the maximum node of the slew bearing is $236.7 \mathrm{Mpa}$. The maximum element equivalent stress is $264.4 \mathrm{Mpa}$. And the allowable stress of $42 \mathrm{CrMo}$ is $310 \mathrm{Mpa}$. So the bolt hole wall thickness to meet the requirements, and has certain space optimization.

\section{Stress analysis of slew bearing's ring}

The stress analysis of the slew bearing's ring is carried out by Solidworks.

The bolt hole ,the top surface of the outer ring, the upper surface of the slew bearing with fixed constraint, and then segmentation of rolling contact ring with rectangular channel, finally each roller has calculated the stress loading to contact one by one rectangle has been good segmentation analysis.

The results of the stress, safety factor and deformation of the finite element calculation of the inner ring raceway are shown in Figure 6.

By analysis

(1)The maximum deformation of the inner ring raceway is $1.299 \mathrm{e}-5(\mathrm{~m})$.

(2)The contact stress is $121 \mathrm{MPa}$;

(3)The end face deformation is $1 \mathrm{e}-33(\mathrm{~m})$.

The results of the stress, safety factor and deformation of the finite element calculation of the outer ring raceway are shown in Figure 7.

By analysis:

(1)The maximum deformation of the weakest upper raceway is $1.902 \mathrm{e}-5(\mathrm{~m})$;

(2) The contact stress is $113 \mathrm{MPa}$;

(3)The deformation of the end face is $1 \mathrm{e}-33(\mathrm{~m})$.

\section{Conclusion}

After analyzing the service occasion, load size and connection mode of slew bearing, the basic structure of slew bearing is designed, and the material, processing mode and heat treatment mode of ring and rolling body are selected. The finite element analysis is carried out by Solidworks and ANSYS, and the following conclusions are obtained. 


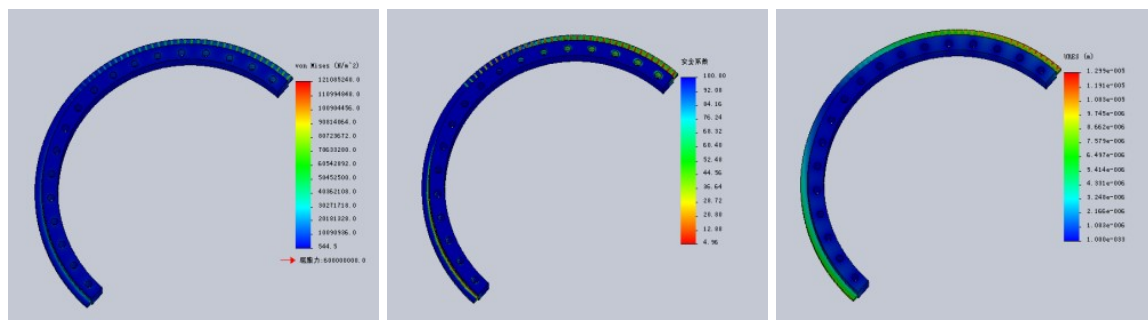

Fig.6 The Figure of Finite Element Analysis for Slew bearing Inner Raceway

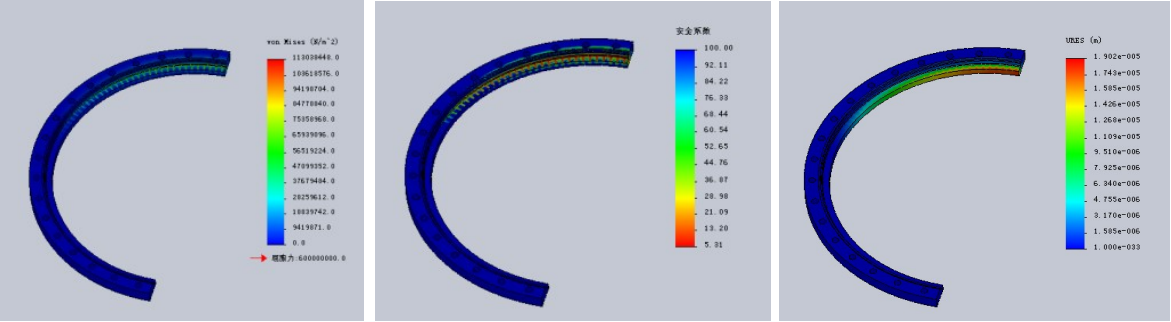

Fig.7 The Figure of Finite Element Analysis for Slew bearing Outer Raceway

(1)According to the requirements of slew bearing, such as service occasion, load size and connection mode, a three row cylindrical roller bearing with the requirement of liquid swevil ring is designed.

(2)The 3D modeling of slew bearing is carried out by using software Solidworks, and the internal and outer ring stresses and the deformation of the end face of the slew bearing are analyzed and calculated. The calculation results show that the contact stress of the inner and outer ring is less than the allowable stress. The deformation of the end face is less than the design requirement, and the deformation amount is $0.005 \mathrm{~mm}$. The stress of the inner and outer rings and the deformation of the end face meet the requirements of the customers, and there is a certain margin of safety.

(3)The stress analysis is carried out on the bolt installation hole of the slew bearing through the ANSYS . Through the analysis, the position and value of the maximum stress are obtained, which provide some reference for the installation, use and maintenance of the slew bearing.

(4)Two kinds of software Solidworks and ANSYS are used to verify the rationality of the design of the bearing and the problems that should be paid attention to in the process of the design of the slew bearing.

(5)The analysis results provide a scientific reference for the design of slew bearing.

\section{Acknowledgements}

This work was financially supported by LiaoNing Mechatronics College(2013017).

\section{References}

[1] Hong Wang, Yuan Chen.Calculation method of bearing capacity and rated life of slew bearing [J].BEARING,2008,(2):7-8.

[2] Shi Shuang Zhang.Research on the functional test of single point mooring (liquid swevil ring) [J].Heavy work and lifting technology ,2012,(4):15-16.

[3] Cheng Gang Fang,Xue Hai Gao,Xiao Diao Huang etc.Design and manufacture of wind power slew bearing [J].BEARING,2009(9):57-62.

[4] ChaoWang, Yi Ning Qu,Ying Qing Ma etc..Liquid swevil ring slew bearing:China,ZL201420795429.X[P].2015-05-13.

[5] Tian Reng Dai,Yan Zhao,Zhao Li Su etc.Selection and installation of common seals for wind power slew bearing [J].BEARING,2012(8):56-58.

[6] ChaoWang,Li Na Zhang,Qiang Hao.The finite element analysis for Slew bearing bolt mounting hole [J].Modern machinery,2015(6):44-45. 